\title{
Spherically symmetric inhomogeneous bianisotropic media: Wave propagation and
} light scattering

Novitsky, Andrey; Shalin, Alexander S.; Lavrinenko, Andrei

Published in:

Physical Review A (Atomic, Molecular and Optical Physics)

Link to article, DOI:

10.1103/PhysRevA.95.053818

Publication date:

2017

Document Version

Publisher's PDF, also known as Version of record

Link back to DTU Orbit

Citation (APA):

Novitsky, A., Shalin, A. S., \& Lavrinenko, A. (2017). Spherically symmetric inhomogeneous bianisotropic media: Wave propagation and light scattering. Physical Review A (Atomic, Molecular and Optical Physics), 95(5), [053818]. https://doi.org/10.1103/PhysRevA.95.053818

\section{General rights}

Copyright and moral rights for the publications made accessible in the public portal are retained by the authors and/or other copyright owners and it is a condition of accessing publications that users recognise and abide by the legal requirements associated with these rights.

- Users may download and print one copy of any publication from the public portal for the purpose of private study or research.

- You may not further distribute the material or use it for any profit-making activity or commercial gain

- You may freely distribute the URL identifying the publication in the public portal 


\title{
Spherically symmetric inhomogeneous bianisotropic media: Wave propagation and light scattering
}

\author{
Andrey Novitsky, ${ }^{1,2}$ Alexander S. Shalin, ${ }^{3}$ and Andrei V. Lavrinenko ${ }^{1}$ \\ ${ }^{1}$ DTU Fotonik, Technical University of Denmark, Ørsteds Plads 343, DK-2800 Kongens Lyngby, Denmark \\ ${ }^{2}$ Department of Theoretical Physics and Astrophysics, Belarusian State University, Nezavisimosti Avenue 4, 220030 Minsk, Belarus \\ ${ }^{3}$ ITMO University, St. Petersburg 197101, Russia \\ (Received 7 March 2017; published 8 May 2017)
}

\begin{abstract}
We develop a technique for finding closed-form expressions for electromagnetic fields in radially inhomogeneous bianisotropic media, both the solutions of the Maxwell equations and material tensors being defined by the set of auxiliary two-dimensional matrices. The approach is applied to determine the scattering cross-sections by spherical particles, the fields inside which correspond to the Airy-exponential waves.
\end{abstract}

DOI: 10.1103/PhysRevA.95.053818

\section{INTRODUCTION}

Closed-form solutions of the Maxwell equations play a tremendous role in electrodynamics. They provide a deep insight into the problem under investigation and their importance cannot be blurred by the development of numerical methods like finite-difference frequency domain (FDFD) technique, finite-difference time domain (FDTD) technique, and finite-element method (FEM). For example, in the area of metamaterials, one often aspires to homogenize a structure to conclude which properties it possesses. Although the wave propagation in such a homogenized material is not catched correctly, its effective material parameters say much about the metamaterial and its properties.

Maxwell's equations have the well-known plane-wave solutions in whichsoever homogeneous media [1-6]. Plane waves of any polarization propagate in the same manner in isotropic media, because the spherical isofrequency surfaces are polarization degenerate. In anisotropic media, the plane waves propagate in different manner depending on their polarization and propagation direction, thus leading to the birefringence and conical refraction [7]. The isofrequency surface in this case is not a sphere anymore, but ellipsoid or hyperboloid [8]. If the medium responds differently on the propagation of rightand left-handed circularly polarized light, it is called chiral, the three-dimensional and planar chirality being introduced [9,10]. Three-dimensional bianisotropic (chiral) material can be described by the Born-Drude-Fedorov constitutive equations $[4,11,12]$. At any rate, there are commonly four plane waves in a homogeneous material (albeit additional waves may appear in spatially dispersive media [13]). Two couples of waves are forward- and backward-propagating. In some degenerate cases the waves in homogeneous bianisotropic media are not plane ones, but the waves with linear, quadratic, or cubic coordinate dependence [14-16].

Periodic materials are the most common inhomogeneous media, because the theoretical background is quite developed either as the Bloch theory for photonic crystals [17] or homogenization for subwavelength metamaterials [18]. However, the truly inhomogeneous media with some continuous dependence of material parameters on coordinates are rarely considered, because there are no general theoretical tools for solving the Maxwell equations except approximate geometrical optics technique [19] and perturbation theory in the case of small inhomogeneity [20]. Transformation optics pushed forward recently provides the elegant solutions of Maxwell equations in inhomogeneous media as well, but for the narrow group of inhomogeneous anisotropic (bianisotropic) materials [21-23]. The strength of transformation optics is in the prediction of the particular designs to conceal and imitate objects, concentrate energy, rotate wavefront, etc. Thus, the restrictions should be imposed on the inhomogeneity, if the analytical solutions are aimed. For example, one can deal with the materials which permittivity function is separable as $\varepsilon(x, y, z)=\varepsilon(x) \varepsilon(y) \varepsilon(z)$. Such materials can be homogenized using the nonlocal approach developed in Ref. [24]. In this paper, we consider the particular case of the materials with factorized characteristics, namely, those possessing one-dimensional inhomogeneity.

One-dimensional inhomogeneity can be realized in the basic geometries as planar, cylindrical, and spherical ones with permittivities $\varepsilon(x), \varepsilon\left(\sqrt{x^{2}+y^{2}}\right)$, and $\varepsilon\left(\sqrt{x^{2}+y^{2}+z^{2}}\right)$, respectively. There are known analytical solutions of Maxwell's equations in linear, parabolic, and exponential planar stratified media [25-27]. Electromagnetic waves have strong nonlocal dispersion and wide-band reflection and transmission [28-30]. Spherically symmetric inhomogeneous media are important in geophysical applications [31] and atmosphere optics [32].

It is important that the Maxwell equations for the waves in stratified media can be solved in the similar manner in Cartesian [33], cylindrical [34], and spherical [35] coordinate systems. Such a unified approach may be applied not only in the way of slicing inhomogeneous medium into the set of thin homogeneous layers, as it was done in Ref. [36] for the calculation of the scattered fields by a cloak, but also for finding the closed-form solutions in the case of the particular distributions of media parameters. The goal of this work is exactly the disclosure of the material parameters allowing analytical solution of the Maxwell equations in inhomogeneous rotationally symmetric bianisotropic media. Applying the scattering theory for inhomogeneous particles, we investigate the scattering properties of the spherical beads characterized by such material tensors. Except for a certain interest for mathematical physics, the analytical solutions may serve for description of structural anisotropy of metamaterials (see recent Refs. [37,38]), caused by the anisotropy of individual meta-atoms but not the lattice. In this case, the multipolar moments of these meta-atoms determine the properties of the whole metamaterial.

The paper consists of six sections. In Sec. II, we outline the matrix approach developed earlier in Ref. [35] for homogeneous media and adapt it for inhomogeneous media. We show that the field differential equations to be solved 
and material tensors are specified by the same set of three two-dimensional matrices $M$. Taking on them appropriately, one can simultaneously solve the Maxwell equations in the closed form and retrieve the material parameters. We also find the limitations on the form of the matrices $M$ imposed by the type of the medium we want to deal with. In Sec. III, we solve the field differential equations for commuting matrices $M$ and obtain arbitrary field profile, as well as Bessel- and Airy-function-based distributions. Noncommuting matrices $M$ are considered in Sec. IV. The example of the preassigned field distribution, subsequent choice of the medium parameters, and calculation of the scattered fields are demonstrated in Sec. V. Section VI concludes the article.

\section{MATRIX APPROACH}

In this section, we generalize the matrix (operator) approach of Ref. [35] to solving Maxwell's equations in inhomogeneous media in spherical coordinates $(r, \theta, \varphi)$. The solutions to be obtained can be straightforwardly applied for calculation of the scattered fields from inhomogeneous particles (see Appendices A and B), though the conventional Mie theory [39-41] can be used as well.

\section{A. Equations for tangential field components}

Monochromatic with circular frequency $\omega$ electric $\mathbf{E}$ and magnetic $\mathbf{H}$ field strengths in bianisotropic media satisfy the Maxwell equations

$$
\begin{aligned}
\nabla \times \mathbf{H}(\mathbf{r}, \omega) & =-i k_{0}[\hat{\varepsilon}(\mathbf{r}, \omega) \mathbf{E}(\mathbf{r}, \omega)+\hat{\alpha}(\mathbf{r}, \omega) \mathbf{H}(\mathbf{r}, \omega)], \\
\nabla \times \mathbf{E}(\mathbf{r}, \omega) & =i k_{0}[\hat{\mu}(\mathbf{r}, \omega) \mathbf{H}(\mathbf{r}, \omega)+\hat{\kappa}(\mathbf{r}, \omega) \mathbf{E}(\mathbf{r}, \omega)],
\end{aligned}
$$

where $\hat{\varepsilon}$ and $\hat{\mu}$ are the dielectric permittivity and magnetic permeability tensors, $\hat{\alpha}$ and $\hat{\kappa}$ are the gyration pseudotensors, $k_{0}=\omega / c$ is the wave number in a vacuum, and $c$ is the speed of light. "Hat" is used only for the three-dimensional tensors.

Throughout the article we consider rotationally symmetric bianisotropic media, which tensorial material parameters are expressed by means of the tensors built up out of the unit vector $\mathbf{e}_{r}=\mathbf{r} / r$ of the spherical coordinate system. There are three such quantities: Dyad $\mathbf{e}_{r} \otimes \mathbf{e}_{r}$ or one-dimensional projector onto the direction $\mathbf{e}_{r}\left[\left(\mathbf{e}_{r} \otimes \mathbf{e}_{r}\right) \mathbf{a}=\mathbf{e}_{r}\left(\mathbf{e}_{r} \mathbf{a}\right)\right.$ and $\mathbf{a}\left(\mathbf{e}_{r} \otimes \mathbf{e}_{r}\right)=$ $\left(\mathbf{a e}_{r}\right) \mathbf{e}_{r}$ for any vector $\left.\mathbf{a}\right]$, two-dimensional projector $\hat{I}=\hat{1}-$ $\mathbf{e}_{r} \otimes \mathbf{e}_{r}$ onto the plane orthogonal to $\mathbf{e}_{r}\left[\hat{I} \mathbf{a}=\mathbf{a}-\left(\mathbf{a} \mathbf{e}_{r}\right) \mathbf{e}_{r}\right.$ for any $\mathbf{a}]$, and tensor $\mathbf{e}_{r}^{\times}$dual to the vector $\mathbf{e}_{r}\left[\mathbf{e}_{r}^{\times} \mathbf{a}=\mathbf{e}_{r} \times \mathbf{a}\right.$ and $\mathbf{a e}_{r}^{\times}=\mathbf{a} \times \mathbf{e}_{r}$ for any $\mathbf{a}$, where $\hat{1}$ is the unit tensor in the three-dimensional space. Then any rotationally symmetric tensor $\hat{\varepsilon}, \hat{\mu}, \hat{\alpha}$, or $\hat{\kappa}$, denoted here as $\hat{\xi}$, can be written as

$$
\hat{\xi}(r, \omega)=\xi_{1}(r, \omega) \mathbf{e}_{r} \otimes \mathbf{e}_{r}+\xi_{2}(r, \omega) \hat{I}+i \chi_{\xi}(r, \omega) \mathbf{e}_{r}^{\times},
$$

where the quantities $\xi_{1}(r, \omega), \xi_{2}(r, \omega)$, and $\chi_{\xi}(r, \omega)$ are the scalar coefficients dependent only on the radial coordinate $r$. To shorten the notation, we drop the frequency dependence in the fields and material tensors further on.

Rotationally symmetric media allow the separation of variables. According to Ref. [35], we may look for the solution of Maxwell's equations in rotationally symmetric media as

$$
\mathbf{E}(\mathbf{r})=\hat{F}_{l m}(\theta, \varphi) \mathbf{E}(r), \quad \mathbf{H}(\mathbf{r})=\hat{F}_{l m}(\theta, \varphi) \mathbf{H}(r)
$$

$\left(\hat{F}_{l m}\right)_{i j}$ is the second rank tensor ( $i$ and $j$ run from 1 to 3 ) expressed through scalar $Y_{l m}(\theta, \varphi)$ and vector $\mathbf{X}_{l m}(\theta, \varphi)=$ $-i[l(l+1)]^{-1 / 2} \mathbf{r} \times \nabla Y_{l m}(\theta, \varphi)$ spherical harmonics as follows:

$$
\hat{F}_{l m}=Y_{l m} \mathbf{e}_{r} \otimes \mathbf{e}_{r}+\mathbf{X}_{l m} \otimes \mathbf{e}_{\theta}+\left(\mathbf{e}_{r} \times \mathbf{X}_{l m}\right) \otimes \mathbf{e}_{\varphi},
$$

where integer numbers $l=0, \ldots, \infty$ and $m=-l,-l+$ $1, \ldots, l-1, l$ are introduced. Unit vectors $\mathbf{e}_{r}, \mathbf{e}_{\theta}$, and $\mathbf{e}_{\varphi}$ are the basis vectors of the spherical coordinates. Tensor functions $\hat{F}_{l m}$ are orthonormalized as

$$
\int_{0}^{\pi} \int_{0}^{2 \pi} \hat{F}_{l^{\prime} m^{\prime}}^{+}(\theta, \varphi) \hat{F}_{l m}(\theta, \varphi) \sin \theta d \theta d \varphi=\hat{1} \delta_{l^{\prime} l} \delta_{m^{\prime} m} .
$$

Here superscript + stands for the Hermitian conjugation. By introducing Eq. (3) into the Maxwell Eqs. (1) and employing the commutation of tensor functions $\hat{F}_{l m}$ with material tensors $\hat{\varepsilon}, \hat{\mu}, \hat{\alpha}$, and $\hat{\kappa}$, we arrive at the system of ordinary differential equations for tangential electric $\mathbf{E}_{t}=\hat{I} \mathbf{E}$ and magnetic $\mathbf{H}_{t}=$ $\hat{I} \mathbf{H}$ fields [35]:

$$
\frac{d \mathbf{W}(r)}{d r}=i k_{0} M(r) \mathbf{W}(r)
$$

where

$$
\begin{aligned}
M & =\left(\begin{array}{cc}
\hat{A} & \hat{B} \\
\hat{C} & \hat{D}
\end{array}\right), \quad \mathbf{W}=\left(\begin{array}{c}
\mathbf{H}_{t} \\
\mathbf{E}_{t}
\end{array}\right), \\
\hat{A} & =\frac{i}{k_{0} r} \hat{I}+\mathbf{e}_{r}^{\times} \hat{\alpha} \hat{I}-\kappa_{1} \delta \frac{l(l+1)}{k_{0}^{2} r^{2}} \mathbf{e}_{\varphi} \otimes \mathbf{e}_{\theta}, \\
\hat{B} & =\mathbf{e}_{r}^{\times} \hat{\varepsilon} \hat{I}-\varepsilon_{1} \delta \frac{l(l+1)}{k_{0}^{2} r^{2}} \mathbf{e}_{\varphi} \otimes \mathbf{e}_{\theta}, \\
\hat{C} & =-\mathbf{e}_{r}^{\times} \hat{\mu} \hat{I}+\mu_{1} \delta \frac{l(l+1)}{k_{0}^{2} r^{2}} \mathbf{e}_{\varphi} \otimes \mathbf{e}_{\theta}, \\
\hat{D} & =\frac{i}{k_{0} r} \hat{I}-\mathbf{e}_{r}^{\times} \hat{\kappa} \hat{I}+\alpha_{1} \delta \frac{l(l+1)}{k_{0}^{2} r^{2}} \mathbf{e}_{\varphi} \otimes \mathbf{e}_{\theta},
\end{aligned}
$$

and $\delta=\left(\varepsilon_{1} \mu_{1}-\alpha_{1} \kappa_{1}\right)^{-1}$. Field strengths $\mathbf{H}=\mathbf{H}_{t}+H_{r} \mathbf{e}_{r}$ and $\mathbf{E}=\mathbf{E}_{t}+E_{r} \mathbf{e}_{r}$ can be written for the known tangential components $\mathbf{H}_{t}$ and $\mathbf{E}_{t}$ as follows:

$$
\begin{aligned}
\left(\begin{array}{l}
\mathbf{H}(r) \\
\mathbf{E}(r)
\end{array}\right) & =V(r)\left(\begin{array}{c}
\mathbf{H}_{t}(r) \\
\mathbf{E}_{t}(r)
\end{array}\right), \\
V & =\left(\begin{array}{cc}
\hat{I}+\frac{\kappa_{1} \delta \sqrt{l(l+1)}}{k_{0} r} \mathbf{e}_{r} \otimes \mathbf{e}_{\theta} & \frac{\varepsilon_{1} \delta \sqrt{l(l+1)}}{k_{0} r} \mathbf{e}_{r} \otimes \mathbf{e}_{\theta} \\
-\frac{\mu_{1} \delta \sqrt{l(l+1)}}{k_{0} r} \mathbf{e}_{r} \otimes \mathbf{e}_{\theta} & \hat{I}-\frac{\alpha_{1} \delta \sqrt{l(l+1)}}{k_{0} r} \mathbf{e}_{r} \otimes \mathbf{e}_{\theta}
\end{array}\right) .
\end{aligned}
$$

\section{B. Equation for $\boldsymbol{\theta}$-components of the fields}

Two types of dependencies on the radial coordinate $r$ can be noticed in the definition of block matrix $M(r)$. They are caused by, first, the inhomogeneity of a bianisotropic medium described by the functions $\xi_{1}(r), \xi_{2}(r)$, and $\chi_{\xi}(r)$ and, second, by the spherical geometry. The latter radial dependence maintains also for homogeneous media. Separating the geometrical and material dependencies, we arrive at

$$
M(r)=M^{(0)}(r)+\frac{1}{r} M^{(1)}+\frac{1}{r^{2}} M^{(2)}(r) .
$$


According to Eq. (7) matrices $M^{(j)}(j=0,1,2)$ can be decomposed in the tensors $\hat{I}, \mathbf{e}_{r}^{\times}$, and $\mathbf{e}_{\varphi} \otimes \mathbf{e}_{\theta}$ of the real three-dimensional space as

$$
\begin{aligned}
& M^{(0)}(r)=M_{\theta \theta}^{(0)}(r) \hat{I}+M_{\varphi \theta}^{(0)}(r) \mathbf{e}_{r}^{\times}, \quad M^{(1)}=\frac{i}{k_{0}} \hat{I}, \\
& M^{(2)}(r)=\frac{l(l+1)}{k_{0}^{2}} M_{\varphi \theta}^{(2)}(r) \mathbf{e}_{\varphi} \otimes \mathbf{e}_{\theta} .
\end{aligned}
$$

Components of decomposition $M_{\theta \theta}^{(0)}=\mathbf{e}_{\theta} M^{(0)} \mathbf{e}_{\theta}, \quad M_{\varphi \theta}^{(0)}=$ $\mathbf{e}_{\varphi} M^{(0)} \mathbf{e}_{\theta}$, and $M_{\varphi \theta}^{(2)}=\mathbf{e}_{\varphi} M^{(2)} \mathbf{e}_{\theta}$ are the $2 \times 2$ matrices determined by the inhomogeneous material parameters:

$$
\begin{aligned}
M_{\theta \theta}^{(0)} & =\left(\begin{array}{cc}
\mathbf{e}_{\theta} \hat{A} \mathbf{e}_{\theta} & \mathbf{e}_{\theta} \hat{B} \mathbf{e}_{\theta} \\
\mathbf{e}_{\theta} \hat{C} \mathbf{e}_{\theta} & \mathbf{e}_{\theta} \hat{D} \mathbf{e}_{\theta}
\end{array}\right)=i\left(\begin{array}{cc}
-\chi_{\alpha} & -\chi_{\varepsilon} \\
\chi_{\mu} & \chi_{\kappa}
\end{array}\right), \\
M_{\varphi \theta}^{(0)} & =\left(\begin{array}{cc}
\alpha_{2} & \varepsilon_{2} \\
-\mu_{2} & -\kappa_{2}
\end{array}\right), \quad M_{\varphi \theta}^{(2)}=\delta\left(\begin{array}{cc}
-\kappa_{1} & -\varepsilon_{1} \\
\mu_{1} & \alpha_{1}
\end{array}\right) .
\end{aligned}
$$

Two-dimensional matrices $M$ can be also presented in terms of their eigenvalues and eigenvectors as

$$
\begin{aligned}
& M_{\theta \theta}^{(0)}(r)=f_{1}(r) \boldsymbol{a}_{1}(r) \otimes \widetilde{\boldsymbol{a}}_{1}(r)+f_{2}(r) \boldsymbol{a}_{2}(r) \otimes \widetilde{\boldsymbol{a}}_{2}(r), \\
& M_{\varphi \theta}^{(0)}(r)=g_{1}(r) \boldsymbol{b}_{1}(r) \otimes \widetilde{\boldsymbol{b}}_{1}(r)+g_{2}(r) \boldsymbol{b}_{2}(r) \otimes \widetilde{\boldsymbol{b}}_{2}(r), \\
& M_{\varphi \theta}^{(2)}(r)=h_{1}(r) \boldsymbol{d}_{1}(r) \otimes \widetilde{\boldsymbol{d}}_{1}(r)+h_{2}(r) \boldsymbol{d}_{2}(r) \otimes \widetilde{\boldsymbol{d}}_{2}(r),
\end{aligned}
$$

where $f_{i}, g_{i}$, and $h_{i}(i=1,2)$ are the eigenvalues of the corresponding matrices. Right and left eigenvectors of a matrix $M$ are defined by the equations $M \boldsymbol{a}_{i}=\lambda_{i} \boldsymbol{a}_{i}$ and $\widetilde{\boldsymbol{a}}_{i} M=\lambda_{i} \widetilde{\boldsymbol{a}}_{i}$, respectively. Orthogonality and normalization conditions for the eigenvectors as $\boldsymbol{a}_{1,2} \widetilde{\boldsymbol{a}}_{2,1}=0$ and $\boldsymbol{a}_{1,2} \widetilde{\boldsymbol{a}}_{1,2}=1$ should be employed.

Equation (6) projected to the directions $\mathbf{e}_{\theta}$ and $\mathbf{e}_{\varphi}$ reads

$$
\begin{aligned}
& \frac{d \boldsymbol{W}_{\theta}}{d r}=i k_{0} M_{\theta \theta} \boldsymbol{W}_{\theta}+i k_{0} M_{\theta \varphi} \boldsymbol{W}_{\varphi}, \\
& \frac{d \boldsymbol{W}_{\varphi}}{d r}=i k_{0} M_{\varphi \varphi} \boldsymbol{W}_{\varphi}+i k_{0} M_{\varphi \theta} \boldsymbol{W}_{\theta},
\end{aligned}
$$

where the bold italic letters denote vectors in the twodimensional space of electric and magnetic components as follows:

$$
\begin{aligned}
& \boldsymbol{W}_{\theta}(r)=\mathbf{e}_{\theta} \mathbf{W}=\left(\begin{array}{c}
\mathbf{e}_{\theta} \mathbf{H} \\
\mathbf{e}_{\theta} \mathbf{E}
\end{array}\right)=\left(\begin{array}{c}
H_{\theta}(r) \\
E_{\theta}(r)
\end{array}\right), \\
& \boldsymbol{W}_{\varphi}(r)=\mathbf{e}_{\varphi} \mathbf{W}=\left(\begin{array}{c}
\mathbf{e}_{\varphi} \mathbf{H} \\
\mathbf{e}_{\varphi} \mathbf{E}
\end{array}\right)=\left(\begin{array}{c}
H_{\varphi}(r) \\
E_{\varphi}(r)
\end{array}\right) .
\end{aligned}
$$

Eliminating the $\varphi$-components of the field vectors from the system of the first-order differential equations one obtains the second-order differential equation for the $\theta$-components of the fields

$$
\begin{aligned}
& \boldsymbol{W}_{\theta}^{\prime \prime}+\left(Q_{1}(r)+\frac{2}{r}\right) \boldsymbol{W}_{\theta}^{\prime} \\
& +\left(Q_{2}(r)+\frac{Q_{1}(r)}{r}+\frac{Q_{3}(r)}{r^{2}}\right) \boldsymbol{W}_{\theta}=0,
\end{aligned}
$$

where prime denotes the derivative over radial coordinate $r$, while the $2 \times 2$ matrices $Q_{1}, Q_{2}$, and $Q_{3}$ take the form

$$
\begin{aligned}
Q_{1}(r)= & -i k_{0}\left(M_{\theta \theta}^{(0)}+M_{\varphi \theta}^{(0)} M_{\theta \theta}^{(0)} M_{\varphi \theta}^{(0)-1}\right)-M_{\varphi \theta}^{(0) \prime}, \\
Q_{2}(r)= & -k_{0}^{2} M_{\varphi \theta}^{(0)}\left(M_{\varphi \theta}^{(0)}+M_{\theta \theta}^{(0)} M_{\varphi \theta}^{(0)-1} M_{\theta \theta}^{(0)}\right) \\
& -i k_{0}\left(M_{\theta \theta}^{(0) \prime}-M_{\varphi \theta}^{(0) \prime} M_{\varphi \theta}^{(0)-1} M_{\theta \theta}^{(0)}\right), \\
Q_{3}(r)= & -l(l+1) M_{\varphi \theta}^{(0)} M_{\varphi \theta}^{(2)} .
\end{aligned}
$$

$\varphi$-components of the fields and, hence, tangential fields can be reconstructed for the known solution $\boldsymbol{W}_{\theta}$ of Eq. (15) as

$$
\mathbf{W}=\boldsymbol{W}_{\theta}(r) \mathbf{e}_{\theta}+\hat{Z} \boldsymbol{W}_{\theta}(r) \mathbf{e}_{\varphi},
$$

where the matrix differential operator $\hat{Z}$ derived from Eq. (13),

$$
\hat{Z}=-M_{\varphi \theta}^{(0)-1}\left(\frac{1}{i k_{0}} \frac{d}{d r}-\frac{i}{k_{0} r}-M_{\theta \theta}^{(0)}\right),
$$

is employed for finding the $\varphi$-components. The full components of the electromagnetic fields follow from Eq. (8).

A straightforward way of finding electromagnetic fields from the Maxwell equations requires carrying out the following steps: first, specify the inhomogeneous distribution of material permittivities, permeabilities, and gyration pseudotensors; second, solve Eq. (15) for the given material parameters; third, find $\mathbf{W}$, i.e., $\mathbf{E}$ and $\mathbf{H}$. However, doing so we have a rare chance to get a solution in the closed form. Instead, we look for the material parameters that provide analytical solutions of Eq. (15). In principle, it could be done as follows. At first we pick up the appropriate matrices $Q_{i}$ that reduce Eq. (15) to an equation with analytical solution. This can be easily done. For the appointed matrices $Q_{i}$ we solve Eq. (16) with respect to matrices $M_{\theta \theta}^{(0)}, M_{\varphi \theta}^{(0)}$, and $M_{\varphi \theta}^{(2)}$. It is the cornerstone issue in such an approach, because it is impossible to find the general closed-form solution of nonlinear differential matrix Eqs. (16) for the given matrices $Q_{i}$. Thus, we need to guess, which functions $Q_{i}(r)$ are appropriate for solving Eq. (15) and simultaneously may result in the solution of Eq. (16).

\section{Material parameters retrieval}

It is always conceivable to retrieve material parameters for the known matrices $M_{\theta \theta}^{(0)}, M_{\varphi \theta}^{(0)}$, and $M_{\varphi \theta}^{(2)}$. Using Eq. (11) the retrieval procedure is straightforward, since each element of the matrices $M$ corresponds to the value $\xi_{1}, \xi_{2}$, or $\chi_{\xi}$. The quantity $\delta$ appears to be the determinant as $\delta=\operatorname{det}\left(M_{\varphi \theta}^{(2)}\right)$. The whole set of material parameters of the rotationally symmetric media then reads

$$
\begin{aligned}
& \hat{\varepsilon}=-\frac{\boldsymbol{e}_{1} M_{\varphi \theta}^{(2)} \boldsymbol{e}_{2}}{\operatorname{det}\left(M_{\varphi \theta}^{(2)}\right)} \mathbf{e}_{r} \otimes \mathbf{e}_{r}+\left(\boldsymbol{e}_{1} M_{\varphi \theta}^{(0)} \boldsymbol{e}_{2}\right) \hat{I}+i\left(\boldsymbol{e}_{1} M_{\theta \theta}^{(0)} \boldsymbol{e}_{2}\right) \mathbf{e}_{r}^{\times}, \\
& \hat{\mu}=\frac{\boldsymbol{e}_{2} M_{\varphi \theta}^{(2)} \boldsymbol{e}_{1}}{\operatorname{det}\left(M_{\varphi \theta}^{(2)}\right)} \mathbf{e}_{r} \otimes \mathbf{e}_{r}-\left(\boldsymbol{e}_{2} M_{\varphi \theta}^{(0)} \boldsymbol{e}_{1}\right) \hat{I}-i\left(\boldsymbol{e}_{1} M_{\theta \theta}^{(0)} \boldsymbol{e}_{2}\right) \mathbf{e}_{r}^{\times}, \\
& \hat{\alpha}=\frac{\boldsymbol{e}_{2} M_{\varphi \theta}^{(2)} \boldsymbol{e}_{2}}{\operatorname{det}\left(M_{\varphi \theta}^{(2)}\right)} \mathbf{e}_{r} \otimes \mathbf{e}_{r}+\left(\boldsymbol{e}_{1} M_{\varphi \theta}^{(0)} \boldsymbol{e}_{1}\right) \hat{I}+i\left(\boldsymbol{e}_{1} M_{\theta \theta}^{(0)} \boldsymbol{e}_{1}\right) \mathbf{e}_{r}^{\times}, \\
& \hat{\kappa}=-\frac{\boldsymbol{e}_{1} M_{\varphi \theta}^{(2)} \boldsymbol{e}_{1}}{\operatorname{det}\left(M_{\varphi \theta}^{(2)}\right)} \mathbf{e}_{r} \otimes \mathbf{e}_{r}-\left(\boldsymbol{e}_{2} M_{\varphi \theta}^{(0)} \boldsymbol{e}_{2}\right) \hat{I}-i\left(\boldsymbol{e}_{2} M_{\theta \theta}^{(0)} \boldsymbol{e}_{2}\right) \mathbf{e}_{r}^{\times},
\end{aligned}
$$


where $\boldsymbol{e}_{1}=(1,0)$ and $\boldsymbol{e}_{2}=(0,1)$. Notice that since the eigenvalues of $M_{\varphi \theta}^{(2)}$ are $h_{1}$ and $h_{2}$, the eigenvalues of matrix $M_{\varphi \theta}^{(2)} / \operatorname{det}\left(M_{\varphi \theta}^{(2)}\right)$ are $h_{2}^{-1}$ and $h_{1}^{-1}$ for the eigenvectors $\boldsymbol{d}_{1}$ and $\boldsymbol{d}_{2}$, respectively. Material tensors Eqs. (18) may realize different types of electromagnetic media. Below we find out the form of matrices $M$ corresponding to different materials.

\section{Lossless bianisotropic media}

Conditions of transparency of bianisotropic media $\hat{\varepsilon}=\hat{\varepsilon}^{+}$, $\hat{\mu}=\hat{\mu}^{+}$, and $\hat{\alpha}=\hat{\kappa}^{+}$are fulfilled, if matrices $M_{\theta \theta}^{(0)}, M_{\varphi \theta}^{(0)}$, and $M_{\varphi \theta}^{(2)} / \operatorname{det}\left(M_{\varphi \theta}^{(2)}\right)$ have the following form in basis of unit vectors $\boldsymbol{e}_{1}$ and $\boldsymbol{e}_{2}$ :

$$
M=\left(\begin{array}{cc}
m_{11}^{\prime}+i m_{11}^{\prime \prime} & m_{12}^{\prime} \\
m_{21}^{\prime} & -m_{11}^{\prime}+i m_{11}^{\prime \prime}
\end{array}\right),
$$

where $m_{11}^{\prime}, m_{11}^{\prime \prime}, m_{12}^{\prime}$, and $m_{21}^{\prime}$ are the real-valued quantities. Such a matrix can be presented in the spectral form as

$$
M=\lambda_{+}(r) \boldsymbol{m}_{+}(r) \otimes \tilde{\boldsymbol{m}}_{+}(r)+\lambda_{-}(r) \boldsymbol{m}_{-}(r) \otimes \tilde{\boldsymbol{m}}_{-}(r),
$$

where $\lambda_{ \pm}=i m_{11}^{\prime \prime} \pm \sqrt{m_{11}^{\prime 2}+m_{12}^{\prime} m_{21}^{\prime}}$ and

$$
\boldsymbol{m}_{ \pm}=\left(\begin{array}{c}
\frac{1}{\sqrt{1+m_{ \pm} n_{ \pm}}} \\
\frac{m_{ \pm}}{\sqrt{1+m_{ \pm} n_{ \pm}}}
\end{array}\right), \quad \widetilde{\boldsymbol{m}}_{ \pm}=\left(\begin{array}{c}
\frac{1}{\sqrt{1+m_{ \pm} n_{ \pm}}} \\
\frac{n_{ \pm}}{\sqrt{1+m_{ \pm} n_{ \pm}}}
\end{array}\right) .
$$

Here, $\quad m_{ \pm}=-\left(m_{11}^{\prime} / m_{12}^{\prime}\right) \pm \sqrt{\left(m_{11}^{\prime} / m_{12}^{\prime}\right)^{2}+\left(m_{21}^{\prime} / m_{12}^{\prime}\right)^{2}}$ and $n_{ \pm}=-\left(m_{11}^{\prime} / m_{21}^{\prime}\right) \pm \sqrt{\left(m_{11}^{\prime} / m_{21}^{\prime}\right)^{2}+\left(m_{12}^{\prime} / m_{21}^{\prime}\right)^{2}}$. If the eigenvectors are constant, then $m_{ \pm}$and $n_{ \pm}$should not depend on $r$. This is achieved for $m_{11}^{\prime}(r)=C_{1} m_{12}^{\prime}(r)$ and $m_{21}^{\prime}(r)=C_{2} m_{12}^{\prime}(r)$ at constant $C_{1}$ and $C_{2}$. Then $m_{ \pm}=-C_{1} \pm \sqrt{C_{1}^{2}+C_{2}^{2}}$ and $n_{ \pm}=-\left(C_{1} \pm \sqrt{C_{1}^{2}+1}\right) / C_{2}$, while $\lambda_{ \pm}(r)=i m_{11}^{\prime \prime}(r) \pm\left|m_{12}^{\prime}(r)\right| \sqrt{C_{1}^{2}+C_{2}}$. If the matrices $M_{\theta \theta}^{(0)}, \quad M_{\varphi \theta}^{(0)}$, and $M_{\varphi \theta}^{(2)} / \operatorname{det}\left(M_{\varphi \theta}^{(2)}\right)$ commute, then the coefficients $C_{1}$ and $C_{2}$ should be the same for each of them.

\section{Nonchiral media}

If $M_{\theta \theta}^{(0)}=0$, the chiral properties of a medium are defined by the pseudogyration tensors $\hat{\alpha}$ and $\hat{\kappa}$, but not by $\chi_{\xi}$. This holds true, when the eigenvalues $f_{i}=0$. If the matrices $M_{\theta \theta}^{(0)}$, $M_{\varphi \theta}^{(0)}$, and $M_{\varphi \theta}^{(2)} / \operatorname{det}\left(M_{\varphi \theta}^{(2)}\right)$ have zeros on the diagonals, the chiral properties are defined by $\chi \xi$.

The medium is anisotropic nonchiral one, if $M_{\theta \theta}^{(0)}=0$, while $M_{\varphi \theta}^{(0)}$ and $M_{\varphi \theta}^{(2)} / \operatorname{det}\left(M_{\varphi \theta}^{(2)}\right)$ are nondiagonal as follows:

$$
M=\left(\begin{array}{cc}
0 & m_{12} \\
m_{21} & 0
\end{array}\right)
$$

The eigenvalues and eigenvectors of such a matrix can be written, respectively, as $\lambda_{ \pm}= \pm \sqrt{m_{12} m_{21}}$ and

$$
\boldsymbol{m}_{ \pm}=\left(\begin{array}{c}
\frac{1}{\sqrt{2}} \\
\pm \sqrt{\frac{m_{21}}{2 m_{12}}}
\end{array}\right), \quad \tilde{\boldsymbol{m}}_{ \pm}=\left(\begin{array}{c}
\frac{1}{\sqrt{2}} \\
\pm \sqrt{\frac{m_{12}}{2 m_{21}}}
\end{array}\right) .
$$

The eigenvectors are constant when $m_{21}(r)=C_{1} m_{12}(r)$. The coordinate dependence is then determined by the eigenvalues $\lambda_{ \pm}(r)= \pm\left|m_{12}(r)\right| \sqrt{C_{1}}$.

\section{Bi-isotropic media}

A bi-isotropic medium characterized by scalar $\varepsilon, \mu, \alpha$, and $\kappa$ requires $M_{\theta \theta}^{(0)}=0$ and the special relations between the other matrices as

$$
\begin{aligned}
M_{\varphi \theta}^{(0)} & =\left(\begin{array}{ll}
m_{11} & m_{12} \\
m_{21} & m_{22}
\end{array}\right), \\
\frac{M_{\varphi \theta}^{(2)}}{\operatorname{det}\left(M_{\varphi \theta}^{(2)}\right)} & =\left(\begin{array}{cc}
m_{22} & -m_{12} \\
-m_{21} & m_{11}
\end{array}\right),
\end{aligned}
$$

matrices $M_{\varphi \theta}^{(0)}$ and $M_{\varphi \theta}^{(2)}$ being commutative. For isotropic media we need to apply $m_{11}=m_{22}=0$.

It can be shown that $M_{\varphi \theta}^{(2)}=\left(M_{\varphi \theta}^{(0)}\right)^{-1}$, i.e., its eigenvalues are the inverse values to those of $M_{\varphi \theta}^{(0)}$. The matrices $Q_{i}$ defined by Eq. (16) have to be as follows:

$$
Q_{1}=-M_{\varphi \theta}^{(0) \prime}, \quad Q_{2}=-k_{0}^{2} M_{\varphi \theta}^{(0) 2}, \quad Q_{3}=-l(l+1) .
$$

Matrix $Q_{3}$ proportional to the two-dimensional unit matrix commutes with the others. $Q_{1}$ and $Q_{2}$ commute, if $M_{\varphi \theta}^{(0)}$ has constant eigenvectors. Another possibility is $M_{\varphi \theta}^{(0) 2}$ is proportional to the two-dimensional identity matrix, while $M_{\varphi \theta}^{(0)}$ does not. It is realized for arbitrary nonconstant eigenvectors and eigenvalues $g_{1}(r)=g(r)$ and $g_{2}(r)=-g(r)$.

\section{SOLUTION OF EQ. (15) FOR COMMUTING MATRICES $M_{\theta \theta}^{(0)}, M_{\varphi \theta}^{(0)}$, AND $M_{\varphi \theta}^{(2)}$}

Let us look at the analytical solutions of Eq. (15) provided the matrices $Q_{1}, Q_{2}$, and $Q_{3}$ commute. Then the vector differential Eq. (15) may be presented as the pair of independent scalar equations. The straightforward way to get the commuting matrices $Q_{i}$ is the usage of the commuting matrices $M_{\theta \theta}^{(0)}, M_{\varphi \theta}^{(0)}$, and $M_{\varphi \theta}^{(2)}$. This means that all matrices $M$ [see Eq. (12)] have the same set of constant right $\boldsymbol{a}_{i}=\boldsymbol{b}_{i}=\boldsymbol{d}_{i}$ and left $\widetilde{\boldsymbol{a}}_{i}=\widetilde{\boldsymbol{b}}_{i}=\widetilde{\boldsymbol{d}}_{i}$ eigenvectors.

Eq. (16) yields

$$
\begin{aligned}
& Q_{1}(r)=-\sum_{i=1}^{2}\left(2 i k_{0} f_{i}+g_{i}^{\prime}\right) \boldsymbol{a}_{i} \otimes \widetilde{\boldsymbol{a}}_{i}, \\
& Q_{2}(r)=-\sum_{i=1}^{2}\left[k_{0}^{2}\left(f_{i}^{2}+g_{i}^{2}\right)+i k_{0} g_{i}\left(\frac{f_{i}}{g_{i}}\right)^{\prime}\right] \boldsymbol{a}_{i} \otimes \tilde{\boldsymbol{a}}_{i}, \\
& Q_{3}(r)=-l(l+1) \sum_{i=1}^{2} g_{i} h_{i} \boldsymbol{a}_{i} \otimes \tilde{\boldsymbol{a}}_{i} .
\end{aligned}
$$

In the scalar equations following from Eq. (15) for commuting matrices Eq. (26), the matrices are replaced with their eigenvalues as $Q_{1} \rightarrow-2 i k_{0} f-g^{\prime}, Q_{2} \rightarrow-k_{0}^{2}\left(f^{2}+g^{2}\right)-$ $i k_{0} g(f / g)^{\prime}$, and $Q_{3} \rightarrow-l(l+1) g h$. Since we have already specified matrices $M$, there is no need to obtain them from the system of nonlinear Eqs. (16). The problem can be now formulated as to determine the closed-form solutions of the differential Eq. (15) for the matrix functions $Q_{i}(r)$ defined by Eq. (26). 
Using the substitution

$$
\boldsymbol{W}_{\theta}(r)=\exp \left\{-\frac{1}{2} \int\left[Q_{1}(r)+\frac{2}{r}-q_{1}(r)\right] d r\right\} \boldsymbol{y}(r)
$$

and commutation of matrices $Q_{1} Q_{2}, Q_{3}, q_{1}$, their derivatives and integrals, Eq. (15) is reduced to

$$
\boldsymbol{y}^{\prime \prime}(r)+q_{1}(r) \boldsymbol{y}^{\prime}(r)+q_{0}(r) \boldsymbol{y}(r)=0,
$$

where $q_{1}$ is an arbitrary matrix and

$$
q_{0}=Q_{2}-\frac{Q_{1}^{2}}{4}-\frac{Q_{1}^{\prime}}{2}+\frac{q_{1}^{2}}{4}+\frac{q_{1}^{\prime}}{2}+\frac{Q_{3}}{r^{2}} .
$$

Exploiting the representations $\boldsymbol{y}(r)=\sum_{i=1}^{2} y_{i}(r) \boldsymbol{a}_{i}$, $q_{0}(r)=\sum_{i=1}^{2} S_{i}(r) \boldsymbol{a}_{i} \otimes \widetilde{\boldsymbol{a}}_{i}$, and $q_{1}(r)=\sum_{i=1}^{2} s_{i}(r) \boldsymbol{a}_{i} \otimes \widetilde{\boldsymbol{a}}_{i}$, we rewrite Eq. (28) as two independent scalar equations. Dropping the index $i=1,2$ in $f_{i}, g_{i}, h_{i}$, and $s_{i}$, we arrive as

$$
y^{\prime \prime}(r)+s(r) y^{\prime}(r)+S(r) y(r)=0,
$$

where

$$
\begin{aligned}
S= & \frac{g^{\prime \prime}}{2}-\frac{g^{\prime 2}}{4}-k_{0}^{2} g^{2}+\frac{i k_{0} f g^{\prime}(2-g)}{2 g}+\frac{s^{2}}{4}+\frac{s^{\prime}}{2} \\
& -\frac{l(l+1) g h}{r^{2}} .
\end{aligned}
$$

\section{A. Arbitrary electromagnetic fields}

Curiously, one may take on any functions $s(r)$ and $S(r)$ in Eq. (30) and then retrieve three functions $f, g$, and $h$ from the single Eq. (31). It is obvious that the functions $f$, $g$, and $h$ are not unique and can be always found. Indeed, for arbitrary $g(r) \neq$ const we can find appropriate $f(r)$ and $h(r)$. It is important that the sought functions $f, g$, and $h$ should not depend on number $l$, because they also set the material tensors, which are $l$-independent. At the same time $S$ depends on $l$ as $S(r)=F_{1}(r)+l(l+1) F_{2}(r)$. This allows us to straightforwardly find the functions $f(r)$ and $h(r)$ for the specified $F_{1}$ and $F_{2}$.

For the known functions $f_{i}(r), g_{i}(r)$, and $h_{i}(r)$ and arbitrary constant vectors $\boldsymbol{a}_{i}$, we can construct matrices $M$ according to Eq. (12). These matrices serve for retrieval of the material tensors using Eq. (18).

A more complicated situation arises when the function $f(r)$ is arbitrary. Then the function $g(r)$ follows from the nonlinear differential equation. But this case is very important, because involved functions $f(r)$, i.e., material parameters $\chi_{\xi}$, may not be achievable in practice, while the simultaneous use of $\chi_{\xi}$ and $\hat{\alpha}, \hat{\kappa}$ makes us to take into account the chirality of a medium twice. Instead of the complicated functions $f(r)$ we may need $f=0$ or $f=$ const. Thus, in the case of arbitrary $f$, the choice $g(r)=$ const delivers several analytical solutions to be discussed below.

\section{B. Bessel solutions}

The vast group of inhomogeneous bianisotropic media admits the solutions of the Maxwell equations in the form of spherical Bessel functions. Naturally, we shall employ the scalar Bessel-like equation following form Eq. (28) at $q_{1}(r)=1 / r$

$$
\begin{gathered}
\boldsymbol{y}^{\prime \prime}(r)+\frac{1}{r} \boldsymbol{y}^{\prime}(r)+\left[Q_{2}(r)-\frac{Q_{1}^{2}(r)}{4}-\frac{Q_{1}^{\prime}(r)}{2}\right. \\
\left.+\frac{1}{r^{2}}\left(Q_{3}(r)-\frac{1}{4}\right)\right] \boldsymbol{y}(r)=0 .
\end{gathered}
$$

For the constant matrices $Q_{i}$, Eq. (32) is apparently Bessel equation. Fields $\boldsymbol{W}_{\theta}=\sqrt{\frac{\pi}{2 r}} \exp \left[-\frac{1}{2} \int Q_{1}(r) d r\right] \boldsymbol{y}(r)$ are in the form of the spherical Bessel functions.

To get the Bessel equation of the form ( $\lambda$ and $m$ are constants)

$$
y^{\prime \prime}+\frac{1}{r} y^{\prime}+\left(\lambda^{2}-\frac{m^{2}}{r^{2}}\right) y=0,
$$

the following equation for the eigenvalues of matrices $Q_{i}$ [see Eq. (26)] should be satisfied:

$$
\begin{aligned}
\frac{g^{\prime \prime}}{2} & -\frac{g^{\prime 2}}{4}-k_{0}^{2} g^{2}+\frac{i k_{0} f g^{\prime}(2-g)}{2 g}-\frac{l(l+1) g h+1 / 4}{r^{2}} \\
& =\lambda^{2}-\frac{m^{2}}{r^{2}}
\end{aligned}
$$

If $g=$ const, then function $f(r)$ is arbitrary. Taking on $h(r)=\left(H_{0}+H_{2} r^{2}\right) / g$ (notice that $H_{0}$ and $H_{2}$ are constants of different dimensions), we find $\lambda^{2}=-k_{0}^{2} g^{2}-l(l+1) H_{2}$ and $m^{2}=l(l+1) H_{0}+1 / 4$.

If $g(r) \neq$ const, then we demand $h(r)=H_{0} / g(r)$. This allows us to find the constant $m^{2}=l(l+1) H_{0}+1 / 4$, while $\lambda^{2}$ is the result of special choice of functions $g(r)$ and $f(r)$ as

$$
\frac{g^{\prime \prime}}{2}-\frac{g^{\prime 2}}{4}-k_{0}^{2} g^{2}+u(r)=\lambda^{2},
$$

where

$$
u(r)=\frac{i k_{0} f g^{\prime}(2-g)}{2 g} .
$$

For arbitrary $g(r)$, function $u(x)=\lambda^{2}-g^{\prime \prime} / 2+g^{\prime 2} / 4+k_{0}^{2} g^{2}$ and, thus,

$$
f(r)=\frac{2 g(r) u(r)}{i k_{0} g^{\prime}(r)[2-g(r)]} .
$$

When we know the whole set of functions $f_{i}(r), g_{i}(r)$, and $h_{i}(r)$, the $\theta$-components of the fields reads

$$
\begin{aligned}
\boldsymbol{W}_{\theta}= & \sum_{i=1}^{2} \exp \left[i k_{0} \int f_{i}(r) d r\right] \\
& \times\left[b_{m_{i}-1 / 2}^{(1)}\left(\lambda_{i} r\right) c_{1}^{(i)}+b_{m_{i}-1 / 2}^{(2)}\left(\lambda_{i} r\right) c_{2}^{(i)}\right] \boldsymbol{a}_{i},
\end{aligned}
$$

where $b_{m-1 / 2}^{(j)}(x)=\sqrt{\frac{\pi}{2 x}} B_{m}^{(j)}(x)$ is the spherical function, $B_{m}^{(j)}(x)$ is the $m$ th-order cylindrical (Bessel, Hankel) function of the kind $j$, and $c_{1,2}^{(i)}$ are the integration coefficients.

For example, let $u_{i}(r)=k_{0}^{2} g_{i}^{2}(r)+g_{i}^{\prime 2}(r) / 4$. Then the solution of Eq. (35) is $g_{i}(r)=\lambda_{i}^{2} r^{2}+a_{1}^{(i)} r+a_{2}^{(i)}$, where $a_{1,2}^{(i)}$ are the arbitrary constants. The remaining functions are equal to

$$
f_{i}=\frac{2 g_{i}\left(k_{0}^{2} g_{i}^{2}+g_{i}^{\prime 2} / 4\right)}{i k_{0} g_{i}^{\prime}\left(2-g_{i}\right)}, \quad h_{i}=\frac{H_{0}^{(i)}+H_{2}^{(i)} r^{2}}{g_{i}} .
$$




\section{Airy and exponential solutions}

A wide class of differential equations corresponds to $q_{1}(r)=0$. In this case, the fields are of the form $\boldsymbol{W}_{\theta}=$ $\frac{1}{r} \exp \left[-\frac{1}{2} \int Q_{1}(r) d r\right] \boldsymbol{y}(r)$, while the differential equation for $\boldsymbol{y}(r)$ reads

$$
\boldsymbol{y}^{\prime \prime}+\left[Q_{2}(r)-\frac{Q_{1}^{2}(r)}{4}-\frac{Q_{1}^{\prime}(r)}{2}+\frac{Q_{3}(r)}{r^{2}}\right] \boldsymbol{y}=0 .
$$

Let us consider solutions for any $f(r)$ in the particular case of $g=$ const. Then the function Eq. (31) turns to

$$
S(r)=-k_{0}^{2} g^{2}-\frac{l(l+1) g h(r)}{r^{2}} .
$$

Using $h(r)=H_{3} r^{3}$ ( $H_{3}$ is constant) one gets the Airy equation $y^{\prime \prime}-x y=0$ with $x(r)=\left[l(l+1) g H_{3}\right]^{1 / 3}\left[r+\frac{k_{0}^{2} g}{l(l+1) H_{3}}\right]$. Hence, the $\theta$-components of the electromagnetic field are equal to

$$
\boldsymbol{W}_{\theta}=\frac{1}{r} \sum_{i=1}^{2} \exp \left[i k_{0} \int f_{i}(r) d r\right]\left[\operatorname{Ai}\left(x_{i}\right) c_{1}^{(i)}+\operatorname{Bi}\left(x_{i}\right) c_{2}^{(i)}\right] \boldsymbol{a}_{i},
$$

where $x_{i}(r)=\left[l(l+1) g_{i} H_{3}^{(i)}\right]^{1 / 3}\left[r+\frac{k_{0}^{2} g_{i}}{l(l+1) H_{3}^{(i)}}\right] . \quad \operatorname{Ai}(x)$ and $\operatorname{Bi}(x)$ are the Airy functions of the first and second kinds.

Another simple solution corresponds to $h(r)=\mathrm{H}_{2} r^{2}$. Then we obtain the oscillator equation $y^{\prime \prime}(r)-k_{0}^{2} \lambda^{2} y(r)=0$ with $\lambda= \pm \sqrt{g^{2}+l(l+1) g H_{2} / k_{0}^{2}}$. Exponential dependence describes either propagating or evanescent fields

$$
\begin{aligned}
\boldsymbol{W}_{\theta}= & \frac{1}{r} \sum_{i=1}^{2} \exp \left[i k_{0} \int f_{i}(r) d r\right] \\
& \times\left[\exp \left(\lambda_{i} k_{0} r\right) c_{1}^{(i)}+\exp \left(-\lambda_{i} k_{0} r\right) c_{2}^{(i)}\right] \boldsymbol{a}_{i},
\end{aligned}
$$

where $\lambda_{i}= \pm \sqrt{g_{i}^{2}+l(l+1) g_{i} H_{2}^{(i)} / k_{0}^{2}}$.

When $g=0$, Eq. (40) has the solution $y(r)=c_{1}+c_{2} r$ for any $f$ and $h$ ( $c_{1}$ and $c_{2}$ are the integration constants). The $\theta$-components of the fields are of the form

$$
\boldsymbol{W}_{\theta}=\sum_{i=1}^{2} \exp \left[i k_{0} \int f_{i}(r) d r\right]\left[\frac{1}{r} c_{1}^{(i)}+c_{2}^{(i)}\right] \boldsymbol{a}_{i} .
$$

Zero material parameters $\varepsilon_{2}=\mu_{2}=\alpha_{2}=\kappa_{2}=0$ can be avoided, if the single eigenvalue $g$ vanishes as $g_{1}=0$ and $g_{2} \neq 0$.

Involved closed-form solutions for $g(r)=$ const and $h(r)=$ $\mathrm{H}_{2} r^{2} \exp (\gamma r)$ stem from the differential equation

$$
y^{\prime \prime}(r)-\left[k_{0}^{2} g^{2}+l(l+1) g H_{2} \mathrm{e}^{\gamma r}\right] y(r)=0
$$

and reads

$$
\begin{aligned}
\boldsymbol{W}_{\theta}= & \sum_{i=1}^{2} \exp \left[i k_{0} \int f_{i}(r) d r\right] \\
& \times\left[I_{\nu_{i}}\left(\lambda_{i} \mathrm{e}^{\gamma^{(i)} r / 2}\right) c_{1}^{(i)}+I_{-v_{i}}\left(\lambda_{i} \mathrm{e}^{\gamma^{(i)} r / 2}\right) c_{2}^{(i)}\right] \boldsymbol{a}_{i},
\end{aligned}
$$

where $v_{i}=2 k_{0} g_{i} / \gamma^{(i)}, \lambda_{i}=2 \sqrt{l(l+1) g_{i} H_{2}^{(i)}} / \gamma^{(i)}$, and $I_{\nu}(x)$ is the modified Bessel function. The solutions of the Maxwell equations in the form of parabolic cylinder and hypergeometric functions can be revealed as well.

\section{SOLUTION OF EQ. (15) FOR NONCOMMUTING MATRICES $M$}

A set of noncommuting matrices $M_{\theta \theta}^{(0)}, M_{\varphi \theta}^{(0)}$, and $M_{\varphi \theta}^{(2)}$ still can result in the commuting matrices $Q_{1}, Q_{2}$, and $Q_{3}$. It is realized when two matrices $Q_{i}$ are proportional to zero or unit matrix and the remaining matrix is arbitrary. For example, $Q_{2}$ and $Q_{3}$ for bi-isotropic media are proportional to the identity matrix, while $Q_{1}$ commutes with them.

In general, it is quite difficult to solve the system of nonlinear Eqs. (16) with respect to matrices $M$. Therefore, we limit ourselves with $M_{\theta \theta}^{(0)}=0$, thus writing

$$
\begin{aligned}
& Q_{1}=-M_{\varphi \theta}^{(0) \prime}, \quad Q_{2}=-k_{0}^{2} M_{\varphi \theta}^{(0) 2}, \\
& Q_{3}=-l(l+1) M_{\varphi \theta}^{(0)} M_{\varphi \theta}^{(2)} .
\end{aligned}
$$

Since $M_{\varphi \theta}^{(0)}$ enters each matrix $Q_{i}$, it is expedient to choose $M_{\varphi \theta}^{(0)}=g \boldsymbol{a}_{1} \otimes \tilde{\boldsymbol{a}}_{1}-g \boldsymbol{a}_{2} \otimes \tilde{\boldsymbol{a}}_{2}$, so that $Q_{1}=0$ and $Q_{2}=-k_{0}^{2} g^{2}$, where $g, \boldsymbol{a}_{i}$, and $\tilde{\boldsymbol{a}}_{i}$ are constant quantities. Arbitrary matrix $Q_{3}$ can be presented in the form

$$
Q_{3}=-l(l+1) \sum_{i=1}^{2} \lambda_{i}(r) \boldsymbol{b}_{i}(r) \otimes \widetilde{\boldsymbol{b}}_{i}(r)
$$

that is

$$
M_{\varphi \theta}^{(2)}=\left(M_{\varphi \theta}^{(0)}\right)^{-1} \sum_{i=1}^{2} \lambda_{i}(r) \boldsymbol{b}_{i}(r) \otimes \widetilde{\boldsymbol{b}}_{i}(r) .
$$

We can exploit the same Eq. (28) for function $\boldsymbol{y}$ only if vectors $\boldsymbol{b}_{i}$ and $\widetilde{\boldsymbol{b}}_{i}$ are constant. We have no strong connection of matrices $M_{\varphi \theta}^{(2)}$ and $M_{\varphi \theta}^{(0)}$ imposed by the commutation at the expense of the very specific matrix $M_{\varphi \theta}^{(0)}$.

For inhomogeneous bi-isotropic media, all matrices $M$ commute, because $M_{\varphi \theta}^{(0)}=\left(M_{\varphi \theta}^{(2)}\right)^{-1}=g(r) \boldsymbol{a}_{1}(r) \otimes \tilde{\boldsymbol{a}}_{1}(r)-$ $g(r) \boldsymbol{a}_{2}(r) \otimes \widetilde{\boldsymbol{a}}_{2}(r)$; therefore, $Q_{2}=-k_{0}^{2} g^{2}(r)$ and $Q_{3}=$ $-l(l+1)$ are proportional to the identity matrix. The remaining matrix $Q_{1}=-M_{\varphi \theta}^{(0) \prime}$ and can be generally presented as

$$
Q_{1}=\sum_{i=1}^{2} \lambda_{i}(r) \boldsymbol{b}_{i} \otimes \widetilde{\boldsymbol{b}}_{i}
$$

where its eigenvalues $\lambda_{i}$ and eigenvectors $\boldsymbol{b}, \widetilde{\boldsymbol{b}}$ can be written in terms of $g(r), \boldsymbol{a}_{i}$, and $\widetilde{\boldsymbol{a}}_{i}$.

\section{AIRY-EXPONENTIAL ELECTROMAGNETIC WAVES IN BIANISOTROPIC MEDIA}

The described approach is applicable not only for inhomogeneous media, but for homogeneous materials as well. In the case of homogeneous isotropic medium with permittivity $\varepsilon$ and permeability $\mu$, one should employ the following eigenvalues and eigenvectors for commuting matrices $M: f_{1}=f_{2}=0$, $g_{1}=-g_{2}=i \sqrt{\varepsilon \mu}, h_{1}=-h_{2}=g_{1}^{-1}$,

$$
\boldsymbol{a}_{1,2}=\left(\begin{array}{c}
\frac{1}{\sqrt{2}} \\
\pm i \sqrt{\frac{\mu}{2 \varepsilon}}
\end{array}\right), \quad \tilde{\boldsymbol{a}}_{1,2}=\left(\begin{array}{c}
\frac{1}{\sqrt{2}} \\
\mp i \sqrt{\frac{\varepsilon}{2 \mu}}
\end{array}\right) .
$$



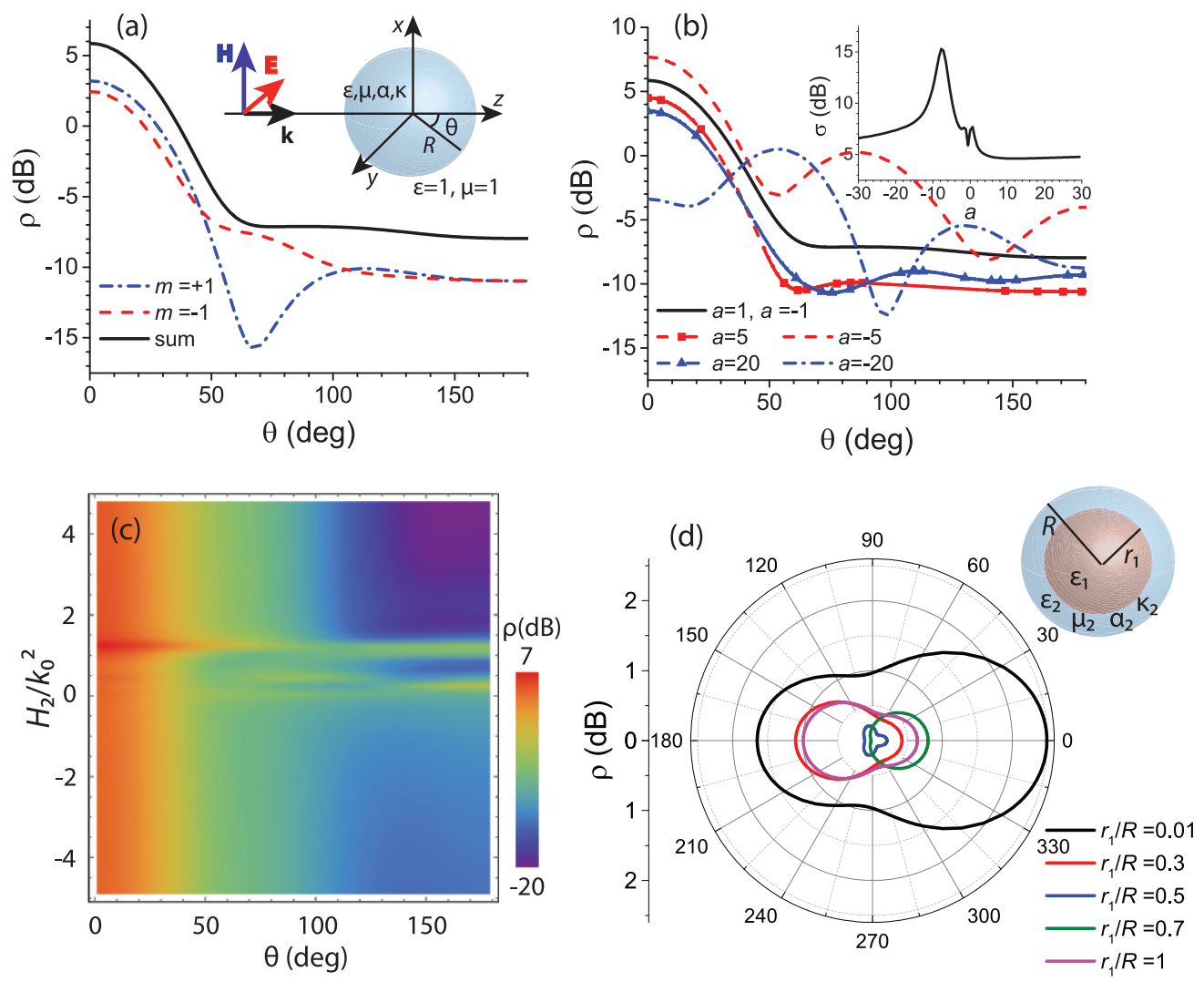

FIG. 1. Scattering cross-sections for the particles supporting Airy-exponential waves. (a) Chirality results in the distinct differential cross-sections $\rho(\theta)$ for the right- and left-handed polarized waves [ $m= \pm 1$ in Eq. (B9)]. The inset sketches geometry of the scattering. Differential cross-section against parameters defining the inhomogeneous medium, quantities (b) $a$ and (c) $H_{2}$. The total cross-section is depicted in the inset in (b). (d) Variation of the differential scattering pattern depending on the radius of the core $r_{1}$ in the core-shell geometry (particle radius $k_{0} R=1$ and core permittivity $\varepsilon_{1}=3.5^{2}$ ). Default parameters: $a=1, g_{1}=1+i, H_{2} / k_{0}^{2}=H_{3} / k_{0}^{3}=1$, and $k_{0} R=\pi$.

The eigenvalues and eigenvectors are expressed by means of the refractive index $\sqrt{\varepsilon \mu}$ and impedance $\sqrt{\mu / \varepsilon}$, respectively.

Let us face the inhomogeneous media now. Being restricted by $M_{\theta \theta}^{(0)}=0$ (or $f_{1}=f_{2}=0$ ) and commutating matrices $M_{\varphi \theta}^{(0)}$ and $M_{\varphi \theta}^{(2)}$, we want one pair of solutions of the Maxwell equations to be described by the Airy functions, whilst another pair by exponentials. As it has been discussed before, the eigenvalues of matrix $M_{\varphi \theta}^{(0)}$ are the constants $g_{1}$ and $g_{2}$. Airy function and exponential demand, respectively, $h_{1}(r)=\mathrm{H}_{3} r^{3}$ and $h_{2}(r)=\mathrm{H}_{2} r^{2}$. Such an ansatz cannot be valid for nonchiral anisotropic media, because in this case the eigenvalues of matrices $M_{\varphi \theta}^{(0)}$ and $M_{\varphi \theta}^{(2)}$ should have opposite signs as $g_{1}=$ $-g_{2}$ and $h_{1}=-h_{2}$. Let us construct arbitrary constant vectors $\boldsymbol{a}_{1,2}$ and $\widetilde{\boldsymbol{a}}_{1,2}$ using the constant parameter $a$ as follows:

$$
\boldsymbol{a}_{1,2}=\frac{1}{\sqrt{2}}\left(\begin{array}{c}
1 \\
\pm a
\end{array}\right), \quad \tilde{a}_{1,2}=\frac{1}{\sqrt{2}}\left(\begin{array}{c}
1 \\
\pm \frac{1}{a}
\end{array}\right)
$$

Thus, the lossy bianisotropic media should possess the tensorial parameters

$$
\begin{aligned}
& \hat{\varepsilon}(r)=-\frac{1}{a^{2}} \hat{\mu}(r)=\frac{1}{a}\left(h_{-}(r) \mathbf{e}_{r} \otimes \mathbf{e}_{r}+g_{-} \hat{I}\right), \\
& \hat{\alpha}(r)=h_{+}(r) \mathbf{e}_{r} \otimes \mathbf{e}_{r}+g_{+} \hat{I}, \\
& \hat{\kappa}(r)=h_{+}(r) \mathbf{e}_{r} \otimes \mathbf{e}_{r}-g_{+} \hat{I},
\end{aligned}
$$

where $g_{ \pm}=\left(g_{1} \pm g_{2}\right) / 2$ and $h_{ \pm}=\left(h_{1}^{-1} \pm h_{2}^{-1}\right) / 2$. We claim lossless bianisotropic medium that can be realized for (i) real $a$ at constant $g_{1}=-g_{2}^{*}=g^{\prime}+i g^{\prime \prime}$ and real functions $h_{1}(r)$ and $h_{2}(r)$, and (ii) imaginary $a$ at imaginary $g_{1}$ and $g_{2}$ and complex functions $h_{1}=-h_{2}^{*}=h^{\prime}+i h^{\prime \prime}$. The Airy-exponential waves can emerge only in the case (i), when the functions $h_{1}$ and $h_{2}$ are arbitrary.

Figure 1 exhibits the result of calculation of the differential scattering cross-section for the particles in free space, which inhomogeneous material distribution is characterized by the real $a$, complex $g_{1}=-g_{2}^{*}$, and real $H_{2}$ and $H_{3}$. There are Airy and exponential waves in such an inhomogeneous spherical particle shown in the inset of Fig. 1(a). Regular solution without singularity at $r=0$ is the superposition of independent solutions as

$$
\begin{aligned}
& T_{11}(r)=\frac{1}{r}\left\{\mathrm{Ai}[x(r)]-\frac{\mathrm{Ai}[x(0)]}{\operatorname{Bi}[x(0)]} \operatorname{Bi}[x(r)]\right\}, \\
& T_{12}(r)=\frac{1}{r}\left[\exp \left(\lambda k_{0} r\right)-\exp \left(-\lambda k_{0} r\right)\right],
\end{aligned}
$$

where $\quad x(r)=\left[l(l+1) g_{1} H_{3}\right]^{1 / 3}\left[r+\frac{k_{0}^{2} g_{1}}{l(l+1) H_{3}}\right] \quad$ and $\lambda=\sqrt{g_{2}^{2}+l(l+1) g_{2} H_{2} / k_{0}^{2}}$. These $T_{11}$ and $T_{12}$ are finite at the limit $r \rightarrow 0$. Using the relationships in Appendix A, one is able to derive the impedance tensor of the wave 
corresponding to the regular solution. Then it can be exploited for determining scattered fields within the matrix scattering theory provided by Appendix B. It is important that the scattering theory for homogeneous particles [35] is similar to that for inhomogeneous beads and differs with only the impedance tensors and evolution operators.

Plane wave linearly polarized as shown in the inset in Fig. 1(a) can be decomposed into a couple of circularly polarized waves of opposite handedness characterized by numbers $m= \pm 1$. Since the scattering particle is chiral $(\hat{\alpha}=$ $\hat{\kappa}^{+} \neq 0$ ), it responds differently to the right- and left-handed waves. In Fig. 1(a), one observes the similar forward- and backward scattering for the waves of opposite handedness and striking difference near the scattering angle $\theta=70^{\circ}$.

The effect of parameter $a$ is demonstrated in Fig. 1(b). The positive values of $a$ result in quite similar dipolarlike distributions of differential cross-section $\rho$ defined by Eq. (B9), while at the negative $a$ the higher-order multipoles are excited. The strongest scattering occurs near $a=-9$ and, in general, it is greater for $a<0$ [see the total cross-section in the inset in Fig. 1(b)]. Changing the ratio between Airy and exponential solutions is the effective control only for the quite small $H_{2} / k_{0}^{2}$ at $H_{3} / k_{0}^{3}=1$ [see Fig. 1(c)]. The backscattering is generally suppressed and for larger $\left|H_{2}\right| / k_{0}^{2}$ it is reduced even more.

When a high-index particle is covered by the inhomogeneous coating, the scattering pattern undergoes the following changes. Without the coating, at $r_{1}=R$, the scattering is mostly backward as demonstrated in Fig. 1(d). With the same overall radius of the particle $R$, the cladding may keep the preferential backscattering $\left(r_{1}=0.3 R\right)$ or result in more forwardly scattered light $\left(r_{1}=0.7 R\right)$. The total scattering is great for the negligible core $\left(r_{1}=0.01 R\right)$ and small for the core radius $R / 2$. In this case the dipole moment is small and the higher-order multipoles come into play (see unusual differential scattering cross-section in this case).

\section{CONCLUSION}

Based on the previous technique for homogeneous materials [35], we have developed the matrix approach to calculate electromagnetic fields in radially inhomogeneous rotationally symmetric bianisotropic media. Important quantities in the approach are the two-dimensional matrices $M_{\theta \theta}^{(0)}(r), M_{\varphi \theta}^{(0)}(r)$, and $M_{\varphi \theta}^{(2)}(r)$. They establish both the form of the field Eqs. (15) and material parameters Eqs. (18). We have revealed the particular forms of matrices $M$ corresponding to the lossless, nonchiral, and bi-isotropic media and demonstrated how the field Eqs. (15) can be solved for commuting and noncommuting matrices $M$. When the matrices $M_{\theta \theta}^{(0)}(r), M_{\varphi \theta}^{(0)}(r)$, and $M_{\varphi \theta}^{(2)}(r)$ are not restricted, one can obtain arbitrary closed-form field distribution (Sec. III A), the medium being generally lossy and bianisotropic. With the restricted matrices $M$ we have found out some particular solutions described by the Bessel, Airy, and exponential functions. These solutions can be mixed, that is, e.g., two solutions out of four are described by Bessel functions and two solutions are Airy functions. Introducing the evolution operators and impedance tensors for inhomogeneous media, we have easily adapted the scattering theory developed in Ref. [35] and applied it to calculation of the Airy-exponential scattered fields and scattering cross-sections.

In what follows, we formulate the advantages of the matrix approach of solution of Maxwell's equations. (i) One is able to find groups of solutions but not particular solutions. The additional freedom is established by the eigenvectors $\boldsymbol{a}_{1,2}$. (ii) The results can be used for different types of media: isotropic, anisotropic, bi-isotropic, and bianisotropic. The more complex material, the richer variety of closed-form solutions. (iii) The solutions for inhomogeneous media can be used in applications (scattering, wave-guiding, etc.) in a similar manner as solutions for homogeneous materials [35]. (iv) Inhomogeneous cylindrically symmetric materials can be considered within the similar approach (Ref. [34] deals with homogeneous media).

Presumably the considered inhomogeneous particles have peculiar optomechanical properties and can be exploited as meta-atoms of artificial structures.

\section{ACKNOWLEDGMENTS}

A.N. acknowledges financial support from the Belarusian Republican Foundation for Fundamental Research (Grant No. F16R-049). A.S. thanks Russian Fund for Basic Research (Project No. 16-52-00112), Russian Science Foundation (Grant No. 16-12-10287) for financial support of the study of scattering cross-sections and patterns, Scholarship of the President of Russian Federation (Grant No. SP-4248.2016.1), and the Ministry of Education and Science of the Russian Federation (GOSZADANIE). A.L. acknowledges partial financial support from Villum Fonden (DarkSILD project).

\section{APPENDIX A: EVOLUTION OPERATORS AND IMPEDANCE TENSORS}

Two basic quantities, evolution operator and impedance tensor, can be constructed grounding on the expressions for the fields. These quantities play an important part in the scattering theory delivered below. Solution of Eq. (15) for the $\theta$-components can be generally written as the sum of four scalar wave solutions $T_{i j}(r)$, integration constants $c_{i}$ and $c_{i}^{\prime}$, and constant vectors $\boldsymbol{a}_{i}$ :

$$
\boldsymbol{W}_{\theta}=\left[T_{11}(r) c_{1}+T_{21}(r) c_{2}\right] \boldsymbol{a}_{1}+\left[T_{12}(r) c_{1}^{\prime}+T_{22}(r) c_{2}^{\prime}\right] \boldsymbol{a}_{2} .
$$

The first index $i=1,2$ in $T_{i j}(r)$ stands for the independent solution of Eq. (15) (e.g., the Bessel functions of the first and second kind), while the second index $j=1,2$ differentiate solutions on the basis of the vector structure of differential Eq. (15). This vector nature of solution exhibits itself in twodimensional vectors $\boldsymbol{a}_{1,2}$.

Tangential components of electromagnetic field defined by Eq. (17) depend on $T_{i j}(r), c_{i}, c_{i}^{\prime}$, and $\boldsymbol{a}_{i}$, too. We will form a couple of constant vectors with the components $c_{i}$ and $c_{i}^{\prime}$ in the tangential plane $(\theta, \varphi)$ as $\mathbf{c}_{1}=c_{1} \mathbf{e}_{\theta}+c_{1}^{\prime} \mathbf{e}_{\varphi}$ and $\mathbf{c}_{2}=$ $c_{2} \mathbf{e}_{\theta}+c_{2}^{\prime} \mathbf{e}_{\varphi}$. Then the solution Eq. (17) for $\mathbf{W}$ can be written in the compact form

$$
\mathbf{W}(r)=\left(\begin{array}{ll}
\hat{\eta}_{1}(r) & \hat{\eta}_{2}(r) \\
\hat{\zeta}_{1}(r) & \hat{\zeta}_{2}(r)
\end{array}\right)\left(\begin{array}{l}
\mathbf{c}_{\mathbf{1}} \\
\mathbf{c}_{2}
\end{array}\right) .
$$


Here $2 \times 2$ matrices $\hat{\eta}$ and $\hat{\zeta}$ define the $r$-dependence of the tangential magnetic and electric fields, respectively. For instance, the tangential magnetic field $\mathbf{H}_{t}=\eta_{1} \mathbf{c}_{1}+\eta_{2} \mathbf{c}_{2}$ is the sum of two independent solutions with the integration constants denoted $\mathbf{c}_{1,2}$. Matrices $\hat{\eta}$ and $\hat{\zeta}$ are ambiguously connected with $T_{i j}, \hat{Z}$, and $\boldsymbol{a}$ as follows:

$$
\begin{aligned}
& \hat{\eta}_{j}(r)=\left(\begin{array}{cc}
\boldsymbol{e}_{1} T_{j 1}(r) \boldsymbol{a}_{1} & \boldsymbol{e}_{1} T_{j 2}(r) \boldsymbol{a}_{2} \\
\boldsymbol{e}_{1} \hat{Z} T_{j 1}(r) \boldsymbol{a}_{1} & \boldsymbol{e}_{1} \hat{Z} T_{j 2}(r) \boldsymbol{a}_{2}
\end{array}\right), \\
& \hat{\zeta}_{j}(r)=\left(\begin{array}{cc}
\boldsymbol{e}_{2} T_{j 1}(r) \boldsymbol{a}_{1} & \boldsymbol{e}_{2} T_{j 2}(r) \boldsymbol{a}_{2} \\
\boldsymbol{e}_{2} \hat{Z} T_{j 1}(r) \boldsymbol{a}_{1} & \boldsymbol{e}_{2} \hat{Z} T_{j 2}(r) \boldsymbol{a}_{2}
\end{array}\right),
\end{aligned}
$$

where the matrices are written in the basis of vectors $\mathbf{e}_{\theta}$ and $\mathbf{e}_{\varphi}$. In the case of homogeneous isotropic media $(\hat{\varepsilon}=\varepsilon \hat{1}, \hat{\mu}=\mu \hat{1}$, and $\hat{\alpha}=\hat{\kappa}=0$ ), one obtains [35]

$$
\begin{gathered}
\hat{\eta}_{j}=g_{l}^{(j)} \mathbf{e}_{\theta} \otimes \mathbf{e}_{\theta}-\frac{i}{\mu k_{0} r} \frac{d\left(r g_{l}^{(j)}\right)}{d r} \mathbf{e}_{\varphi} \otimes \mathbf{e}_{\varphi}, \\
\hat{\zeta}_{j}=g_{l}^{(j)} \mathbf{e}_{\theta} \otimes \mathbf{e}_{\varphi}+\frac{i}{\varepsilon k_{0} r} \frac{d\left(r g_{l}^{(j)}\right)}{d r} \mathbf{e}_{\varphi} \otimes \mathbf{e}_{\theta},
\end{gathered}
$$

where $g_{l}^{(j)}\left(k_{0} r \sqrt{\varepsilon \mu}\right)=T_{j 1}=T_{j 2}$ is the solution in the form of spherical Bessel or Hankel functions of the first $(j=1)$ and second $(j=2)$ kind.

Matrix representation of Eq. (A2) is advantageous for writing evolution operators and impedance tensors. Evolution operator $\Omega_{r_{0}}^{r}$ serves for finding tangential fields $\mathbf{W}(r)$ at some point $r$, if the fields $\mathbf{W}\left(r_{0}\right)$ at the point $r_{0}$ are known, by means of the relationship $\mathbf{W}(r)=\Omega_{r_{0}}^{r} \mathbf{W}\left(r_{0}\right)$. Writing Eq. (A2) for $r$ and $r_{0}$ and excluding the constant vector $\left(\mathbf{c}_{1}, \mathbf{c}_{2}\right)^{T}$, we arrive at the evolution operator,

$$
\Omega_{r_{0}}^{r}=\left(\begin{array}{ll}
\hat{\eta}_{1}(r) & \hat{\eta}_{2}(r) \\
\hat{\zeta}_{1}(r) & \hat{\zeta}_{2}(r)
\end{array}\right)\left(\begin{array}{ll}
\hat{\eta}_{1}\left(r_{0}\right) & \hat{\eta}_{2}\left(r_{0}\right) \\
\hat{\zeta}_{1}\left(r_{0}\right) & \hat{\zeta}_{2}\left(r_{0}\right)
\end{array}\right)^{-1},
$$

where the inversion of the $4 \times 4$ matrix should be performed.

Equation (A5) is applicable only in the spatial regions where the whole set of solutions exists (spherical layer). However, if a solution has a singularity at some point (usually at $r=0$ or $r=\infty$ ), it should be eliminated. In this case we need to isolate the independent solutions in Eq. (A2). Setting $\mathbf{c}_{2}=0\left(\mathbf{c}_{1}=0\right)$ yields the first (second) solution as follows:

$$
\begin{aligned}
& \mathbf{W}^{(1)}(r)=\left(\begin{array}{l}
\mathbf{H}_{t 1} \\
\mathbf{E}_{t 1}
\end{array}\right)=\left(\begin{array}{l}
\hat{\eta}_{1} \mathbf{c}_{1} \\
\hat{\zeta}_{1} \mathbf{c}_{1}
\end{array}\right)=\left(\begin{array}{l}
\hat{\eta}_{1}(r) \\
\hat{\zeta}_{1}(r)
\end{array}\right) \mathbf{c}_{1}, \\
& \mathbf{W}^{(2)}(r)=\left(\begin{array}{l}
\mathbf{H}_{t 2} \\
\mathbf{E}_{t 2}
\end{array}\right)=\left(\begin{array}{l}
\hat{\eta}_{2} \mathbf{c}_{2} \\
\hat{\zeta}_{2} \mathbf{c}_{2}
\end{array}\right)=\left(\begin{array}{l}
\hat{\eta}_{2}(r) \\
\hat{\zeta}_{2}(r)
\end{array}\right) \mathbf{c}_{2} .
\end{aligned}
$$

Constant vectors $\mathbf{c}_{1,2}$ for independent waves can be expressed in terms of the known magnetic fields as follows $\mathbf{H}_{t 1}\left(r_{0}\right)=$ $\hat{\eta}_{1}\left(r_{0}\right) \mathbf{c}_{1}$ and $\mathbf{H}_{t 2}\left(r_{0}\right)=\hat{\eta}_{2}\left(r_{0}\right) \mathbf{c}_{2}$.

Each independent wave $j=1,2$ is characterized by the impedance tensor $\hat{\Gamma}_{j}$ defined through $\mathbf{E}_{t j}=\hat{\Gamma}_{j} \mathbf{H}_{t j}$. Using Eq. (A6), we get

$$
\hat{\Gamma}_{j}(r)=\hat{\zeta}_{j}(r) \hat{\eta}_{j}^{-1}(r)
$$

\section{APPENDIX B: SCATTERING THEORY}

In principle, there are two basic geometries deserving consideration. The first one is the entirely inhomogeneous particle of radius $R$. In this case, we should properly choose the solution $T_{i j}$ to exclude field singularity at the center. The second geometry can be named "homogeneous core""inhomogeneous shell," any set of independent solutions $T_{i j}$ being possible in the shell. Let us start with the general multilayer geometry of the particle assuming we have $N$ spherical layers with material tensors $\hat{\xi}^{(j)}$, each expands from $r_{j-1}$ to $r_{j}\left(r_{0}=0\right.$ and $\left.r_{N}=R\right)$. Incident field characterized by the strengths $\mathbf{H}_{\text {inc }}(\mathbf{r})$ and $\mathbf{E}_{\text {inc }}(\mathbf{r})$ in free space is scattered by such a particle generating the fields

$$
\begin{aligned}
{\left[\begin{array}{c}
\mathbf{H}_{\mathrm{sc}}(\mathbf{r}) \\
\mathbf{E}_{\mathrm{sc}}(\mathbf{r})
\end{array}\right]=} & \sum_{l=0}^{\infty} \sum_{m=-l}^{l}\left[\begin{array}{cc}
\hat{F}_{l m}(\theta, \varphi) & 0 \\
0 & \hat{F}_{l m}(\theta, \varphi)
\end{array}\right] \\
& \times V_{\mathrm{sc}}^{l}(r)\left[\begin{array}{c}
\hat{I} \\
\widetilde{\Gamma}^{l}(r)
\end{array}\right] \widetilde{\eta}^{l}(r)\left(\widetilde{\eta}^{l}(R)\right)^{-1} \mathbf{H}_{\mathrm{sc}}^{l m}(R),
\end{aligned}
$$

where $\widetilde{\eta}$ and $\widetilde{\zeta}$ are described in terms of spherical Hankel functions of the first kind $h_{v}^{(1)}(x), \widetilde{\Gamma}^{l}(r)=\widetilde{\zeta}^{l}(r)\left[\widetilde{\eta}^{l}(r)\right]^{-1}$ is the impedance tensor of the scattered wave, and $\mathbf{H}_{\mathrm{sc}}^{l m}(R)$ is the tangential magnetic field of a spherical wave $(l, m)$ at particle's interface. Surface impedance tensor at the particle interface can be written as $\widetilde{\Gamma}^{l}=\widetilde{\zeta}^{l}(R)\left[\widetilde{\eta}^{l}(R)\right]^{-1}$. It should be noted that we intentionally omit the "hat" over the tensors with tildes on top.

Since the tangential fields are continuous at the interfaces between the multiple cocentric spherical layers, we are able to write $\mathbf{W}(R)=\Omega_{r_{N-1}}^{R} \mathbf{W}\left(r_{N-1}\right), \mathbf{W}\left(r_{N-1}\right)=\Omega_{r_{N-2}}^{r_{N-1}} \mathbf{W}\left(r_{N-2}\right), \ldots$, $\mathbf{W}\left(r_{2}\right)=\Omega_{r_{1}}^{r_{2}} \mathbf{W}\left(r_{1}\right)$, where $\mathbf{W}$ is the field inside the spherical particle. Hence, the tangential fields at the outer interface $\mathbf{W}(R)=\Omega_{r_{1}}^{R} \mathbf{W}\left(r_{1}\right)$ are expressed by means of the evolution operator of the whole set of spherical layers defined as $\Omega_{r_{1}}^{R}=\Omega_{r_{N-1}}^{R} \Omega_{r_{N-2}}^{r_{N-1}} \ldots \Omega_{r_{1}}^{r_{2}}$. Tangential fields at the first interface $r=r_{1}$ are defined by the surface impedance tensor $\hat{\Gamma}_{1}=$ $\hat{\zeta}_{1}\left(r_{1}\right) \hat{\eta}_{1}^{-1}\left(r_{1}\right)$ of the inner sphere $0<r \leqslant r_{1}$ (we need to consider only the nonsingular solution $j=1$ ). Thus, the field at the outer interface reads

$$
\begin{aligned}
{\left[\begin{array}{l}
\mathbf{H}(R, \theta, \varphi) \\
\mathbf{E}(R, \theta, \varphi)
\end{array}\right]=} & \sum_{l=0}^{\infty} \sum_{m=-l}^{l}\left[\begin{array}{cc}
\hat{F}_{l m}(\theta, \varphi) & 0 \\
0 & \hat{F}_{l m}(\theta, \varphi)
\end{array}\right] \\
& \times V_{N}^{l}(R) \Omega_{r_{1}}^{R}\left(\begin{array}{c}
\hat{I} \\
\hat{\Gamma}_{1}^{l}
\end{array}\right) \mathbf{H}_{1}^{l m}\left(r_{1}\right),
\end{aligned}
$$

where $V_{N}$ is the matrix Eq. (8) in the $N$ th layer and $\mathbf{H}_{1}^{l m}\left(r_{1}\right)$ are the tangential magnetic fields of spherical waves $(l, m)$ at the interface $r=r_{1}$.

The boundary conditions claim continuity of the tangential fields as

$$
\mathbf{W}_{\mathrm{inc}}(R, \theta, \varphi)+\mathbf{W}_{\mathrm{sc}}(R, \theta, \varphi)=\mathbf{W}(R, \theta, \varphi) .
$$

Multiplication of this equation by the Hermitian conjugate matrix $\hat{F}_{l^{\prime} m^{\prime}}^{+}(\theta, \varphi)$, integration over the angles $\theta$ and $\varphi$, and 
exploiting Eq. (5) yields

$$
\mathbf{W}_{\mathrm{inc}}^{l m}+\left(\begin{array}{c}
\hat{I} \\
\widetilde{\Gamma}^{l}
\end{array}\right) \mathbf{H}_{\mathrm{sc}}^{l m}(R)=\Omega_{r_{1}}^{R}\left(\begin{array}{c}
\hat{I} \\
\hat{\Gamma}_{1}^{l}
\end{array}\right) \mathbf{H}_{1}^{l m}\left(r_{1}\right)
$$

where

$$
\begin{aligned}
\mathbf{W}_{\mathrm{inc}}^{l m} & =\int_{0}^{\pi} \int_{0}^{2 \pi} \hat{F}_{l m}^{+}(\theta, \varphi) \mathbf{W}_{\mathrm{inc}} \sin \theta \mathrm{d} \theta \mathrm{d} \varphi \\
& =\int_{0}^{\pi} \int_{0}^{2 \pi}\left[\begin{array}{c}
\hat{F}_{l m}^{+}(\theta, \varphi) \hat{I} \mathbf{H}_{\mathrm{inc}}(R, \theta, \varphi) \\
\hat{F}_{l m}^{+}(\theta, \varphi) \hat{I} \mathbf{E}_{\mathrm{inc}}(R, \theta, \varphi)
\end{array}\right] \sin \theta \mathrm{d} \theta \mathrm{d} \varphi .
\end{aligned}
$$

The solution of Eq. (B4) results in the scattering amplitude

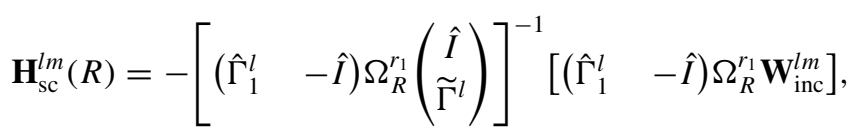

where $\Omega_{R}^{r_{1}}=\left(\Omega_{r_{1}}^{R}\right)^{-1}$ is the inverse evolution operator.

When there is only the core $(N=1)$, the evolution operator $\Omega_{R}^{R}$ turns to the four-dimensional identity matrix and the scattering amplitudes are of the form

$$
\mathbf{H}_{\mathrm{sc}}^{l m}(R)=\left(\widetilde{\Gamma}^{l}-\hat{\Gamma}_{1}^{l}\right)^{-1}\left[\left(\begin{array}{ll}
\left(\hat{\Gamma}_{1}^{l}\right. & -\hat{I}) \mathbf{W}_{\mathrm{inc}}^{l m}
\end{array}\right] .\right.
$$

Having in mind that at $r \rightarrow \infty$, the spherical Hankel function $h_{l}^{(1)}\left(k_{0} n^{(0)} r\right)$ equals $i^{-l-1} e^{i k_{0} n^{(0)} r} / r$, we derive the scattered fields in the far zone

$$
\left(\begin{array}{l}
\mathbf{H}_{\mathrm{sc}}(\mathbf{r}) \\
\mathbf{E}_{\mathrm{sc}}(\mathbf{r})
\end{array}\right)=\frac{1}{r} e^{i k_{0} n^{(0)} r}\left(\begin{array}{c}
\mathbf{e}_{\theta} \otimes \mathbf{e}_{\theta}+\frac{1}{\gamma^{(0)}} \mathbf{e}_{\varphi} \otimes \mathbf{e}_{\varphi} \\
\mathbf{e}_{\theta} \otimes \mathbf{e}_{\varphi}-\gamma^{(0)} \mathbf{e}_{\varphi} \otimes \mathbf{e}_{\theta}
\end{array}\right) \sum_{l=0}^{\infty} \sum_{m=-l}^{l} i^{-l-1} \hat{F}_{l m}(\theta, \varphi)\left[\widetilde{\eta}^{l}(R)\right]^{-1} \mathbf{H}_{\mathrm{sc}}^{l m}(R),
$$

where $n^{(0)}=\sqrt{\varepsilon^{(0)} \mu^{(0)}}$ and $\gamma^{(0)}=\sqrt{\mu^{(0)} / \varepsilon^{(0)}}$ are the refractive index and impedance for ambient medium. Impedance tensor Eq. (A7) at infinity equals $\widetilde{\Gamma}=-\gamma^{(0)} \mathbf{e}_{r}^{\times}$.

Differential scattering cross-section normalized to the geometrical cross-section of the particle $\sigma_{g}=\pi R^{2}$ shows which part of energy comes into the solid angle $d o$ and equals $\frac{d \sigma}{\sigma_{g} d o}=\frac{r^{2}}{\pi R^{2}} \frac{\left|\mathbf{H}_{\mathrm{cc}}\right|^{2}}{\left|\mathbf{H}_{\text {inc }}\right|^{2}}$. Integrating (averaging) over the azimuthal angle $\varphi$ results in the cross-section $\rho(\theta)=\frac{d \sigma}{\sigma_{g} \sin \theta d \theta}$ dependent on the scattering angle $\theta$ as

$$
\rho(\theta)=\frac{1}{\pi R^{2}\left|\mathbf{H}_{\mathrm{inc}}\right|^{2}} \sum_{m=-\infty}^{\infty}\left|\sum_{l=|m|}^{\infty} i^{-l-1} \hat{F}_{l m}(\theta, 0)\left[\widetilde{\eta}^{l}(R)\right]^{-1} \mathbf{H}_{\mathrm{sc}}^{l m}(R)\right|^{2} .
$$

Integrating differential cross-section over all directions, one arrives at the total scattering cross-section

$$
\frac{\sigma}{\sigma_{g}}=\frac{1}{\pi R^{2}\left|\mathbf{H}_{\mathrm{inc}}\right|^{2}} \sum_{l=0}^{\infty} \sum_{m=-l}^{l}\left|\left[\tilde{\eta}^{l}(R)\right]^{-1} \mathbf{H}_{\mathrm{sc}}^{l m}(R)\right|^{2}
$$

[1] M. Born and E. Wolf, Principles of Optics (Cambridge University Press, Cambridge, 1997).

[2] L. D. Landau, E. M. Lifshitz, and L. P. Pitaevskii, Electrodynamics of Continuous Media (Butterworth-Heinemann, Oxford, 2004).

[3] F. I. Fedorov, Optics of Anisotropic Media (Izdatelstvo Akademii Nauk BSSR, Minsk, 1958).

[4] F. I. Fedorov, Theory of Gyrotropy (Nauka i Tehnika, Minsk, 1976).

[5] A. N. Serdyukov, I. V. Semchenko, S. A. Tretyakov, and A. H. Sihvola, Electromagnetics of Bi-anisotropic Materials (Gordon and Breach Sciense Publishers, Amsterdam, 2001).

[6] T. G. Mackay and A. Lakhtakia, Electromagnetic Anisotropy and Bianisotropy: A Field Guide (World Scientific, Singapore, 2010).

[7] A. Turpin, Y. V. Loiko, T. K. Kalkandjiev, and J. Mompart, Conical refraction: Fundamentals and applications, Laser Photon. Rev. 10, 750 (2016).

[8] A. Poddubny, I. Iorsh, P. Belov, and Y. Kivshar, Hyperbolic metamaterials, Nat. Photon. 7, 948 (2013).
[9] C. M. Soukoulis and M. Wegener, Past achievements and future challenges in the development of three-dimensional photonic metamaterials, Nat. Photon. 5, 523 (2011).

[10] V. A. Fedotov, P. L. Mladyonov, S. L. Prosvirnin, A. V. Rogacheva, Y. Chen, and N. I. Zheludev, Asymmetric Propagation of Electromagnetic Waves through a Planar Chiral Structure, Phys. Rev. Lett. 97, 167401 (2006).

[11] P. Drude, Lehrbuch der Optik (Verlag von S. Hirzel, Leipzig, 1912).

[12] M. Born, Optik (J. Springer, Heidelberg, 1933).

[13] V. M. Agranovich and V. Ginzburg, Crystal Optics with Spatial Dispersion and Excitons (Springer, Berlin, 1984).

[14] G. N. Borzdov, Evolutionary operators of electromagnetic waves in crystals. I. Classification: Waves with cubic dependence of amplitude on coordinates, Sov. Phys. Crystallogr. 35, 313 (1990).

[15] G. N. Borzdov, Evolutionary operators of electromagnetic waves in crystals. II. Waves with linear dependence of amplitude on coordinates, Sov. Phys. Crystallogr. 35, 317 (1990). 
[16] G. N. Borzdov, Evolutionary operators of electromagnetic waves in crystals. III. Waves with quadratic dependence of amplitude on coordinates, Sov. Phys. Crystallogr. 35, 322 (1990).

[17] J. D. Joannopoulos, S. G. Johnson, J. N. Winn, and R. D. Meade, Photonic Crystals: Molding the Flow of Light (Princeton University Press, Princeton, 2008).

[18] C. R. Simovski, On electromagnetic characterization and homogenization of nanostructured metamaterials, J. Opt. 13, 013001 (2011).

[19] Yu. A. Kravtsov and Yu. I. Orlov, Geometrical Optics of Inhomogeneous Media (Springer-Verlag, Berlin, 1990).

[20] G. P. Agrawal and C. L. Mehta, Angular spectrum approach to electromagnetic wave propagation in inhomogeneous media, Opt. Commun. 14, 88 (1975).

[21] J. B. Pendry, D. Schurig, and D. R. Smith, Controlling electromagnetic fields, Science 312, 1780 (2006).

[22] U. Leonhardt and T. G. Philbin, Geometry and light: The science of invisibility, Prog. Opt. 53, 69 (2009).

[23] A. V. Novitsky, S. V. Zhukovsky, L. M. Barkovsky, and A. V. Lavrinenko, Field approach in the transformation optics concept, Prog. Electromagn. Res. 129, 485 (2012).

[24] A. Ciattoni, D. Rago, and C. Rizza, Separable metamaterials: Analytical ab initio homogenization and chirality, J. Opt. 18, 115103 (2016).

[25] D. R. Hartree, Optical and equivalent paths in a stratified medium treated from a wave standpoint, Proc. R. Soc. London A 131, 428 (1931).

[26] O. Rydbeck, The reflection of electremagnetic waves from a parabolic ionized layer, Philos. Mag. 34, 342 (1943).

[27] J. R. Wait, Electromagnetic Waves in Stratified Media (Pergamon Press, Oxford, 1970).

[28] A. B. Shvartsburg, Dispersion of electromagnetic waves in stratified and non-stationary media (exactly solvable models), Phys. Usp. 43, 1201 (2000).

[29] A. B. Shvartsburg and A. A. Maradudin, Waves in Gradient Metamaterials (World Scientific, Singapore, 2013).
[30] J. Lekner, Theory of Reflection: Reflection and Transmission of Electromagnetic, Particle, and Acoustic Waves (Springer, Heidelberg, 2016).

[31] L. N. Frazer, Use of the spherical layer matrix in inhomogeneous media, Geophys. J. R. Astron. Soc. 50, 743 (1977).

[32] V. A. Babenko, L. G. Astafyeva, and V. N. Kuzmin, Electromagnetic Scattering in Disperse Media: Inhomogeneous and Anisotropic Particles (Springer-Verlag, Berlin, 2003).

[33] L. M. Barkovskii, G. N. Borzdov, and A. V. Lavrinenko, Fresnel's reflection and transmission operators for stratified gyroanisotropic media, J. Phys. A: Math. Gen. 20, 1095 (1987).

[34] A. V. Novitsky and L. M. Barkovsky, Operator matrices for describing guiding propagation in circular bianisotropic fibres, J. Phys. A: Math. Gen. 38, 391 (2005).

[35] A. Novitsky and L. Barkovsky, Matrix approach for light scattering from a multilayered rotationally symmetric bianisotropic sphere, Phys. Rev. A 77, 033849 (2008).

[36] C.-W. Qiu, A. Novitsky, H. Ma, and S. Qu, Electromagnetic interaction of arbitrary radial-dependent anisotropic spheres and improved invisibility for nonlinear-transformation-based cloaks, Phys. Rev. E 80, 016604 (2009).

[37] K. L. Koshelev and A. A. Bogdanov, Interplay between anisotropy and spatial dispersion in metamaterial waveguides, Phys. Rev. B 94, 115439 (2016).

[38] V. Kozlov, D. Filonov, A. S. Shalin, B. Z. Steinberg, and P. Ginzburg, Asymmetric backscattering from the hybrid magnetoelectric meta particle, Appl. Phys. Lett. 109, 203503 (2016).

[39] C. F. Bohren and D. R. Huffman, Absorption and Scattering of Light by Small Particles (John Wiley and Sons, New York, 1983).

[40] L. Tsang, J. A. Kong, and K.-H. Ding, Scattering of Electromagnetic Waves: Theories and Applications (John Wiley and Sons, New York, 2000).

[41] C.-W. Qiu, L.-W. Li, T.-S. Yeo, and S. Zouhdi, Scattering by rotationally symmetric anisotropic spheres: Potential formulation and parametric studies, Phys. Rev. E 75, 026609 (2007). 